\title{
El docente, el currículo y la familia frente a la violencia ${ }^{1}$
}

\author{
Helena Morales Ortega \\ helena.morales@uac.edu.co \\ Universidad Autónoma del Caribe
}

\section{Resumen}

Ante la violencia que acusa a la sociedad y, especialmente en la escuela, existe la necesidad de replantear y reorientar la labor de la familia y la escuela, como escenarios socializadores, con la labor central del docente y de la mano con un currículo que propicie una cultura escolar de paz, sana convivencia, tolerancia y sin discriminación. En este sentido, este texto propone realizar un análisis crítico desde la familia como eje de enseñanzas o disvalores, desde la escuela como escenario socializador en que se manifiestan las diferentes expresiones culturales de la sociedad, especialmente con estereotipos que perviven en el imaginario colectivo, y desde la violencia escolar, caso particular de la violencia general.

\section{Palabras clave}

Violencia escolar; currículo; cultura escolar de paz; imaginario colectivo; escenario socializador;

Recibido 17/02/2020-Aceptado 23/04/2020

\footnotetext{
${ }^{1}$ Este capítulo es resultado del proyecto de investigación titulado "Violencia y victimización en tres escuelas del distrito de Barranquilla: Una mirada desde la normatividad de la protección integral del niño, niña y adolescente" desarrollado dentro del Grupo de Investigación Estudios de Género, Niñez y Criminalidad.
} 


\title{
The teacher, the curriculum, and the family against violence
}

\begin{abstract}
The violence has been given that stands within society and, especially in school, there is a need to rethink and reorient the work of the family and the school, as socializing scenarios, with the central work of the teacher and hands on a curriculum that fosters a school culture of peace, healthy coexistence, tolerance and without discrimination. In this sense, this text proposes to carry out a critical analysis from the family as the axis of teachings or disvalues, from the school as a socializing scenario in which the different cultural expressions of society are manifested, especially with stereotypes that survive in the collective imagination, and from school violence particular case within general violence.
\end{abstract}

\section{Keywords}

School violence, curriculum, school culture of peace, collective imagination, socializing scenario 


\section{O professor, o currículo e a família contra a violência}

\section{Resumo}

A violência que culpou nossa sociedade e, principalmente na escola, é necessário repensar e reorientar o trabalho da família e da escola, como cenários de socialização, com o trabalho central do professor e de mãos dadas com um currículo que promova um cultura escolar de paz, convivência saudável, tolerância e sem discriminação. Nesse sentido, este texto propõe realizar uma análise crítica da família como eixo de ensinamentos ou desvalorizações, da escola como cenário de socialização no qual se manifestam as diferentes expressões culturais da sociedade, principalmente com estereótipos que sobrevivem na imaginação coletiva; e da violência escolar, um caso particular de violência geral. 


\section{Introducción}

La escuela como escenario socializador, constituye un espacio donde se manifiestan las diferentes expresiones culturales de la sociedad, por lo tanto, ella reproduce igualmente los estereotipos que sobre los géneros perviven en el imaginario colectivo. Las cifras de violencia en la sociedad, y particularmente en las familias colombianas, hace que muchos actores de la sociedad e investigadores se cuestionan sobre las posibles soluciones al problema y plantean que la respuesta se podría encontrar en la escuela; en la educación que se imparte y la que se debería impartir (Chaux y Velásquez, 2004). Se espera que los estudiantes vayan al colegio a relacionarse con los demás y que las instituciones educativas les enseñen a hacerlo. Instrucción y educación son procesos donde la escuela juega un papel fundamental, valores y actitudes para vivir en armonía social, son temas que se supone atraviesan toda la educación que reciben los estudiantes.

En este sentido, se reconoce que a través de los tiempos la escuela ha prestado un servicio social a la comunidad, siendo el lugar propicio para la formación de los individuos que ingresan por primera vez a un mundo externo, desconocido hasta ese momento por ellos. En ese preciso momento el niño o la niña dejan de pertenecer exclusivamente a la familia, como el contexto donde se desarrolla moral y éticamente (Castro, 2006, p. 46). Costumbres, valores, principios, buenos modales, comportamientos, respeto, entre otras muchas significaciones, son aprendidos en la escuela.

En este nuevo escenario, primeramente, aparecen los maestros y guardadores o cuidadores, quienes se convertirán en figuras de autoridad, mientras estén en la escuela, pero también serán estos los que transformen su vida e influirán - se espera - de manera positiva en su desarrollo. En otras palabras, los profesores jugarán un papel determinante para la configuración y conformación del tipo de persona que la sociedad necesita para responder a los nuevos retos, cambios y paradigmas en una sociedad también cambiante. Se ha afirmado (Carrillo, 2009), que los efectos más relevantes de la escolarización no son precisamente aquellos relacionados con el aprendizaje de los contenidos programáticos, académicos, sino aquellos que pueden considerarse como efectos colaterales o secundarios del sistema académico; estos son realmente importantes en la definición de las transformaciones de los modos de sentir, pensar y actuar de los estudiantes por cuanto son mucho más duraderos y relacionados con la construcción de la personalidad. 
En segundo lugar, aparecen los pares u otros alumnos, quienes también se constituirán en piezas claves para el desarrollo de las relaciones interpersonales, y es precisamente en esas relaciones que los jóvenes empiezan a tener diferencias, ya sea por la religión que profesan, por sus hábitos o costumbres, o bien sea por su temperamento, y es a partir de ese instante que comienzan los conflictos y en muchos casos la violencia escolar, la cual se manifiesta de muchas maneras desde la violencia psicológica, emocional y hasta física. Como bien lo sintetiza Fernández

[...] La escuela es una organización altamente reglada, con todo tipo de convenciones, valores y procedimientos, marcados todos ellos por el modo de hacer cotidiano. También se nutre de formas standard para la distribución de espacios, de tiempos, de agrupaciones de alumnos [...] Esto supone que aquellos alumnos cuyas motivaciones o valores personales no encajan con el formato de escuela o con los intereses y conocimientos que allí se comunican, representan un problema escolar y, en un caso social (2007, p. 17-18).

En consecuencia, los sistemas y las instituciones educativas tienen un papel protagónico y un compromiso con el cambio social, puesto que desde la escuela es posible estimular las transformaciones sociales, siempre y cuando docentes y directivos construyan paradigmas que contemplen formas de interacción solidaria, participativa, consensual y respetuosa, necesarias para edificar una sociedad pluralista, justa y equitativa; como también un currículo enfocado al no conflicto y a una sana convivencia. De esta manera, se hablaría de una educación centrada en la persona, y una escuela que se convierte en un espacio de desarrollo social y convivencia armónica.

Sin lugar a dudas, este es un aspecto muy importante a tener en cuenta para comprender la problemática de la violencia y la convivencia escolar, pues es la labor que el maestro cumple en el aula, y más específicamente el "poder" que tiene éste: hoy aún en muchas escuelas él es quien tiene el control y manejo absoluto de la clase. El maestro es quien ordena, quien da las instrucciones, indica la estrategia didáctica que utilizará, la meta o el propósito que quiere lograr durante el desarrollo de la clase; es él la máxima autoridad, inmerso en una concepción tradicional de la educación, donde el estudiante no tiene derecho de hablar, solo escuchar en silencio. En ocasiones el ejercicio del poder de directivos y maestros, se sustenta más en prácticas represivas e intolerantes — que generan temor en el estudiante y no respeto 
basado en la comprensión e interiorización de la importancia del cumplimiento de los reglamentos de la escuela y la norma en general.

A este respecto, ese no es el papel que le otorga la educación a quien enseña; por el contrario, tal como lo afirma Fernández:

En un cierto sentido, el control del aula es un elemento clave para prevenir posibles perturbaciones. Cómo se realice ese control es el tema que se debate en este texto. También hay que ser cautos y cuidadosos de no caer en la posibilidad de que sea el control en sí mismo el objetivo de la educación, en cuyo caso se convierte en sumisión por parte del alumnado, como sostiene uno de los argumentos más reñidos y que con más fuerza se arguye contra este enfoque. En este caso el control se convierte en un impedimento para el aprendizaje (2011, p.146).

Relacionado con lo anterior, Díaz-Aguado (2004) en su investigación realizada en España, con adolescentes y adultos, encontró que estos, frecuentemente se ven inmersos en conflictos y no los resuelven de manera inteligente, sino que ponen en riesgo la tranquilidad y obstaculizan no solo el bienestar de sus víctimas sino también su propio bienestar, porque con su violencia aumentan (por lo menos a medio y largo plazo) las tensiones y conflictos que originaron la conducta violenta. Tal es el caso de Manuel (nombre cambiado para proteger su privacidad ), un alumno de cuarto de la E.S.O ${ }^{2}$ de 16 años, en la entrevista realizada en dicha investigación, al preguntarle por los problemas en el instituto, tratando de evaluar, cómo percibe él su propia situación expresó (antes de participar en los programas prevención de la violencia):

Me han echado de clase muchas veces por enfrentarme a algunos profesores. [...] $\mathrm{Me}$ faltan al respeto. Abusan de ser profesores. Como el otro día una profesora me dijo que la clase estaba mejor sin mí y yo le dije que si estaba mejor sin mí entonces también estaba mejor sin ella. [...] Si me porto mal y hay unas normas que me las expliquen [...] podrían intentar hablar conmigo, llevarme por otro camino. [...] son profesores. Ellos sabrán cómo, pero no diciéndome esas cosas porque me incitan a que me ponga nervioso y empeore todo. Esta clase es problemática. Todos lo dicen. Nos hemos juntado la mayoría repetidores. [...] este año he empezado mal y no creo que tenga solución. ¿Si tú fueras director del instituto qué harías para resolver estas situaciones? Cambiar el modo de dar la clase y echando al alumno o al profesor. Porque a mí me expulsan, pero a ellos no (Díaz-Aguado, 2004, p.94).

${ }^{2}$ E.S.O = Escuela secundaria obligatoria. 
Este caso refleja también la realidad que se vive en muchas escuelas del país donde los docentes "blindados" (no se sabe de qué), irrespetan a sus alumnos sin tener en cuenta el papel preponderante que juegan no solo en la formación académica de éstos, sino en la parte axiológica, que privilegia la formación integral de los adolescentes. Así no es como se concibe la educación. Por lo contrario, la educación es uno de los principales caminos para avanzar hacia el cambio social. Visto lo anterior,

[...] el profesor se convierte en un facilitador del conocimiento, que motiva y ayuda a desarrollar las capacidades de los alumnos teniendo en cuenta sus características personales y aportándoles una atención y trabajo personal que se adapte a sus necesidades y posibilidades de éxito y de aprendizaje (Fernández, 2011, p.145).

En el mismo sentido, Pérez sostiene que:

[...] los seres humanos somos producto del sistema social, a su vez el sistema social es producto nuestro según como se vaya constituyendo la cotidianidad en los diversos y múltiples espacios de interacción [...] la educación por sí misma no puede transformar a la sociedad, pero no hay cambios sociales efectivos si no está presente la educación en ellos (2014, p.31).

Por ello, buena parte de lo que ocurra en el futuro del país depende de lo que hagan o no los educadores, por lo tanto, su gestión es fundamental para que los alumnos comprendan el mundo desde diversos lenguajes, aprendan a vivir con los demás y sean productivos. No quiere decir esto que los docentes son los culpables de la violencia en las escuelas; de ninguna manera, por el contrario, lo que se pretende es destacar el valor de los profesores y de la escuela en el papel transformador que les otorga la sociedad y, por supuesto, confirmar su participación en la función social, axiológica y en las distintas formas de relación para una práctica sana de la convivencia escolar. De ahí que, cuando se piensa que un profesor es un formador de seres humanos y que muchas veces es el profesional que pasa más tiempo con los niños y jóvenes, se hace evidente la importancia social de su trabajo y las diversas responsabilidades de su profesión. El maestro contemporáneo se enfrenta a retos que le impone su disciplina, y a otros que le demanda la sociedad. Hoy en día, el docente requiere de competencias diferentes para solucionar con creatividad los primeros, y participar así en la solución de los segundos. Eso sí, para que los maestros puedan lograr un trabajo óptimo, 
es necesario realizarlo en conjunto con la familia, y, a la vez, el docente debe dar un espacio a los alumnos en donde se genere la confianza, la democracia, diálogo e interacción, es decir, un ambiente físico y emocional excelente.

\section{Diseño metodológico}

En nuestro estudio, al preguntarle a los estudiantes acerca de la disposición de sus profesores a ayudarles, un 52,5\% respondió que estos se mostraban muy dispuestos, el 36.6\% manifestó que los docentes se mostraban más bien dispuestos, contra un 7,8\% que señaló que sus profesores se mostraban más bien no dispuestos a ayudarles y un 3\% afirmó que los maestros nunca estaban dispuestos a ayudarlos ( Ver Gráfica $1^{\circ}$ ).

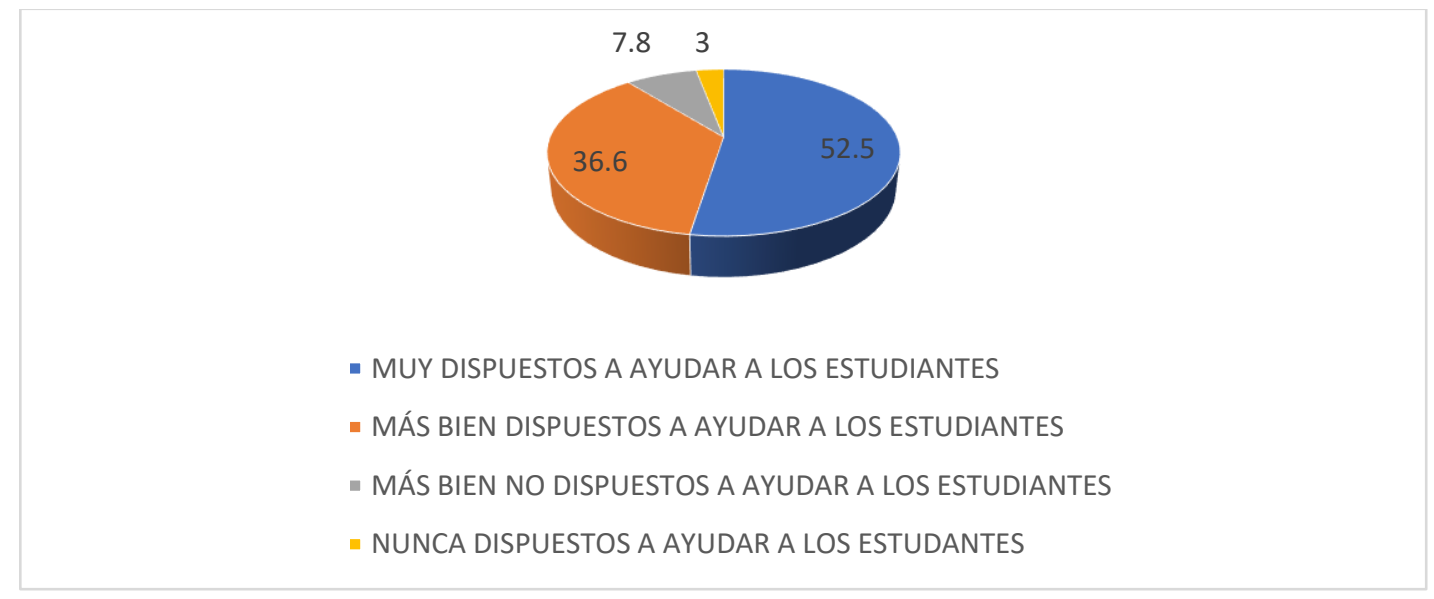

\section{Grafica 10. Disposición de ayuda por parte de los profesores}

Fuente: Elaboración propia

Preguntado el estudiante acerca de la percepción de la calidad de las relaciones entre él/ella y sus profesores, se encontró que un alto porcentaje, un 50,9\%, expresó que eran muy buenas y el 37,2\% manifestó que las consideraban más bien buenas. Solo el 11,9\% consideró que eran malas o más bien malas (Ver Gráfica $2^{\circ}$ ). 


\section{¿Cómo consideras que son las relaciones entre tú y los} profesores en tu clase?

9.32 .6

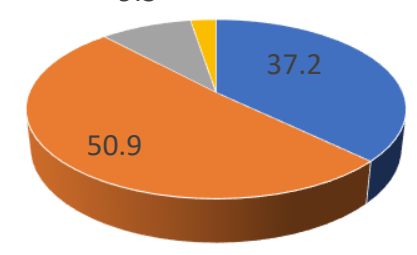

- MUY BUENAS - MÁS BIEN BUENAS " MÁS BIEN MALAS " MUY MALAS

\section{Grafica 2. Percepción de la calidad de las relaciones entre los estudiantes y sus profesores.}

Fuente: Elaboración propia

Entonces, para hacer que la escuela se convierta en un espacio de interacción y desarrollo social, se requiere de docentes, además de comprometidos, formados para la solución de conflictos que se puedan presentar en las instituciones educativas. En tal sentido, afirma Pérez

ejercitar su poder transformador requiere que el docente amplíe su capacidad de acción en los espacios escolares, de modo que complemente la labor de transmisión de conocimientos disciplinares - que es una tarea específica al campo de formación profesional como docente, ya sea en matemáticas, química, inglés, español, física, etc. - para asumir también una función formativa frente a los estudiantes en cuanto a su cosmovisión, a las formas de ver e interactuar en los entornos social y natural - que es una tarea transversal que compete a todos los educadores sin distingo del área disciplinar en la que se desempeñen (2014a, p. 31).

Por otra parte, Martínez (2014) dice:

[...] para lograr la adecuada resolución pacífica de los conflictos, es preciso que, en la institución, al menos, se den los siguientes pasos: a) que se reconozca el conflicto como parte integrante de la vida escolar; b) que se capacite a todo el personal directivo, administrativo y de profesores en el manejo adecuado de esto; c) que se establezcan criterios y procedimientos de resolución de los mismos [...] (p. 60). 
Ahora bien, frente al problema de la violencia en la escuela, la ignorancia que se puede tener frente al tema hace que no se vea, con lo cual se puede negar, con el consecuente olvido del sufrimiento de las víctimas, lo cual constituye el peor escenario. Pero también es preocupante cuando los profesores detectan un caso y lo manejan en la forma menos adecuada, esto es, trivializándolo o sugiriéndole al niño agredido que es su responsabilidad. Se trata, como lo afirma Hoyos (2005), de una doble victimización, pues además de estar sufriendo por el maltrato, al niño agredido se le culpa por la situación. Otros piensan que estas conductas son formadoras del carácter y la personalidad, razón por la cual las dejan pasar o sugieren que si les pegan lo mejor es devolver el golpe, lo cual dificulta la educación en una cultura de la no violencia. La escuela, como institución que forma, está llamada a estimular la construcción de espacios de solidaridad, diálogo y concertación, a brindar los espacios y oportunidades que permitan el ejercicio de los derechos de los niños, a propiciar una cultura de respeto y de sana convivencia entre los estamentos que la conforman. Los profesores/as y los padres tienen una responsabilidad especial en el cuidado de niños/as y adolescentes y eso incluye ayudar a quien está siendo víctima de conductas no deseadas en la escuela. En todas las estructuras encargadas de acoger los niños, es responsabilidad de cada educador poner en obra los derechos de éstos, igualmente de informarles de ellos.

La escuela es un lugar privilegiado, la mayoría de los niños pasan por ella y el docente es el agente activo que está más tiempo en contacto con los menores de edad, lo que le permite observar el comportamiento del alumno y la interacción con compañeros. Al transformarse en un testigo del desarrollo socio-emocional e intelectual del niño, adquiere gran importancia para los profesionales de la educación poseer formación que les ayude a detectar los casos de niños que sean maltratados, conocer las mejores estrategias de intervención, qué hacer ante el niño, cómo dirigir la entrevista con los padres, a dónde dirigirse en caso de que tengan que intervenir agentes especializados, etc. (Castro, 2006. p 46).

Es responsabilidad de cada educador poner en obra los derechos de los niños, brindarles la protección integral entendida ésta como

[...] el reconocimiento que se le hace a los niños-(as) y adolescentes como sujetos de derechos, la garantía y cumplimiento de los mismos, la prevención de su amenaza o vulneración y la seguridad de su restablecimiento inmediato en desarrollo del principio del interés superior. La protección integral se materializa en el conjunto de políticas, 
programas y acciones que se ejecuten en los ámbitos nacional, departamental, distrital y municipal con la correspondiente asignación de recursos financieros, físicos y humanos (Artículo 7 ley 1098 de 2006).

\section{Currículo y violencia en la escuela}

El fenómeno de la violencia escolar se ha convertido en uno de los problemas psicológicos y sociales más serios que viven los niños, las niñas y los adolescentes en las instituciones de educación básica primaria y secundaria; y sin duda alguna, también es una de las peores experiencias que viven los docentes, rectores y coordinadores de las instituciones educativas. En este orden de ideas, se puede indicar que el tema del presente estudio ha sido mirado desde diferentes perspectivas. Sin embargo, el interés de esta parte se centra básicamente en realizar un análisis crítico de la violencia escolar desde la estructura curricular, y, en particular, desde el campo pedagógico, porque es desde allí desde el cual se define el tipo de ser humano que las instituciones educativas se proponen formar.

En consonancia con este análisis, se hace necesario realizar algunas precisiones sobre el currículo, para lo cual este se entiende como:

[...] el conjunto de criterios, planes de estudio, programas, metodologías, y procesos que contribuyen a la formación integral y a la construcción de la identidad cultural nacional, regional y local, incluyendo también los recursos humanos, académicos y físicos para poner en práctica las políticas y llevar a cabo el proyecto educativo institucional (Artículo 76, ley 115 de 1994).

Al respecto, Tyler, R. (1976, p 5) indica que el currículo "son todas las experiencias de aprendizaje que los alumnos obtienen como producto de planificación, orientación y supervisión de la Institución educacional, sean éstas individuales o grupales, como dentro o fuera de la escuela, en pos de determinados fines educativos”. Para Arnaz el currículo es

[...] el plan que norma y conduce explícitamente un proceso concreto y determinante de enseñanza - aprendizaje que se desarrolla en una institución educativa [...] Es un conjunto interrelacionado de conceptos, proposiciones y normas, estructurado en forma anticipada a acciones que se quiere organizar; en otras palabras, es una construcción conceptual destinada a conducir acciones, pero no las acciones mismas, si bien de ellas se desprenden evidencias que hacen posible introducir ajustes o modificaciones al plan (1995, p.72). 
Johnson considera que "el currículo es algo más que el conjunto de las experiencias del aprendizaje" (1967, p.13); se refiere al aprendizaje terminal del alumno como resultado de la enseñanza. Para este autor, el currículo especifica los resultados que se desean obtener del aprendizaje, los cuales deben estar estructurados previamente; de esta manera hace referencia a los fines como resultado del aprendizaje y sostiene que el currículo no establece los medios -es decir, las actividades y los materiales- sino los fines. Por su parte, De Arredondo (1981), citado por Díaz Barriga lo concibe como:

[...] el resultado de: a) el análisis y reflexión sobre las características del contexto, del educando y de los recursos; b) la definición (tanto explícita como implícita) de los fines y los objetivos educativos; c) la especificación de los medios y los procedimientos propuestos para asignar racionalmente los recursos humanos, materiales, informativos, financieros, temporales y organizativos de manera tal que se logren los fines propuestos (2008, p.20).

Todas estas acepciones hacen referencia al llamado «currículum explícito», el cual, tradicionalmente, se ha manifestado en un documento que contiene de forma expresa toda la planificación de las actividades que la escuela realiza, los objetivos, los contenidos, metodologías, cronograma, etc. Pero se conoce que además de este currículo expreso con sus contenidos y valores, que persigue objetivos educacionales, existe en simultánea el llamado currículo oculto, a través del cual la escuela, y particularmente el docente, de una forma tácita, enseñanza más que conceptos, saber hacer o procedimiento relacionados con su área de enseñanza o conocimiento, transmite una educación en valores, entrega "normas sociales y expectativas que tiene la sociedad sobre los/las estudiantes [...] es decir, que las escuelas hacen lo que se supone que deben hacer; dicho de otra forma, entregan lo que será útil para el orden social establecido (Apple \& King, [1977], citado por Sandoval [ 2014, p.152]).

Al respecto, Carrillo, dice que:

[...] podemos entender el currículo oculto, como aquel que hace referencia a los conocimientos, destrezas, valores, actitudes y normas que se adquieren en los procesos de enseñanza-aprendizaje $y$, en general, en todas las interacciones que se dan cotidianamente en el aula y la escuela, pero que no llegan a explicitarse como metas educativas a lograr de una mera intencionada. Frente al currículo explícito que se 
desarrolla en las escuelas, este otro es de carácter invisible, pero no por ello deja de actuar de manera eficaz en el proceso educativo existente entre alumnos y alumnas y profesores y profesoras $(2009$, p.2).

Entre las características del currículo oculto se ha señalado que es omnipresente porque se encuentra presente o actúa en todos los momentos de la vida académica; es omnímodo porque se expresa de diferentes maneras y su influencia es múltiple; también es reiterativo a través de las actividades que se repiten rutinariamente; es invaluable, es decir que sus repercusiones en múltiples ocasiones no son valoradas.

Sandoval sobre este, afirma que,

[...] a través del currículum oculto se transmiten las expectativas asociadas al papel de los/las estudiantes (sumisión, obediencia, etcétera), las cuales entran en contradicción con los objetivos del currículum explícito (autonomía, capacidad crítica), expectativas, que con frecuencia resultan imposibles de entender para determinados estudiantes, a los que con frecuencia se denomina como "conflictivos" (2014, p.156).

Jackson (2001), citado por Sandoval destaca tres aspectos básicos del currículum oculto existente en la escuela tradicional, que él señala que conducen al aprendizaje de la sumisión y la heteronomía. Ellos son:

La monotonía de la vida escolar. Los/las estudiantes deben aprender a esperar, a tener paciencia, a permanecer inmóviles, durante largos periodos de tiempo, ignorando a sus compañeros.

La naturaleza de la evaluación educativa: Su carácter no explícito, sus contradicciones y la imposibilidad de que los estudiantes puedan discutirla. Los/las estudiantes deben aprender cómo funciona este complejo mecanismo para garantizarse el máximo de recompensas y el mínimo de castigos, aprender a acomodarse a las expectativas de los demás y conseguir la aprobación simultánea de dos audiencias, el profesor y los compañeros, que con frecuencia entran en conflicto.

La fuerte jerarquización de la vida escolar y la concentración del control en el profesor: Los/las estudiantes deben acostumbrarse a la diferencia de poder, sustituyendo los propios planes e iniciativas por los que el profesor (a) impone (2014, p.156). 
¿Y cómo se transmite este currículum oculto? Se transfiere de manera inconsciente a través de las interacciones sociales que se establecen en la escuela, de ahí la gran importancia del docente, pues en su trato de los estudiantes, en su vocabulario, etc., se expresa este elemento importante de la formación del educando.

No es posible abarcar todos los hechos, fenómenos, dinámicas, todo lo que ocurre en el espacio escolar, pero lo que sí queda establecido es que el llamado currículo oculto constituye una poderosa herramienta que bien usada puede servir para promover el cambio social, para enseñar a los educandos valores y actitudes que les brinden herramientas para el pensamiento crítico y autocrítico frente a un mundo cada vez más cambiante.

Precisado lo anteriormente expuesto, señalamos que Abraham Magendzo, analiza la relación existente entre convivencia escolar y currículo así:

[...] al vincular la convivencia escolar con el currículum se está, por un lado, preguntando por cuáles son los aprendizajes que se debieran intencionarse deliberadamente con el fin de promover la convivencia escolar, por el otro, nos desafía a interrogar la cultura escolar con el fin de tomar conciencia de cuáles son los mensajes ocultos que desde ella se están enviando en relación con la convivencia escolar. Se suma a esto, la necesidad de indagar y cuestionar por qué ciertos aprendizajes vitales para la convivencia escolar como son los relacionados, por ejemplo, con la educación en derechos humanos, educación para la paz, educación para la resolución de conflictos en términos pacíficos, educación para la tolerancia y la no-discriminación, educación para la intersubjetividad y la alteridad, tienen escaso poder, legitimidad y presencia en el currículo (2003, p.1).

Esta cita sugiere replantear y reorientar el currículo de las escuelas de todo el país hacia una cultura escolar de la paz, la sana convivencia, la tolerancia; el no sexismo, una educación sin discriminación a todos los niños, niñas y jóvenes de Colombia con el fin de reafirmar los valores de los educandos. Detrás de ello, podría emplearse también la propuesta presentada por Arias Sanabria en el texto denominado "Conocimiento didáctico del contenido de la enseñanza de ética y valores humanos, un estudio de caso", el cual se sintetiza "una transformación del contenido“ mediante una comprensión de la "ética y valores humanos”, mediatizada por una práctica del CDC (contenido, evaluación, estrategias de enseñanza, finalidades de enseñanza, conocimiento sobre los estudiantes y contexto), aplicada a una práctica pedagógica de la enseñanza de la ética y valores en el que se evidencia como 
finalidad del docente el fomento de la autoestima como habilidad para la vida (2019, pp. 514).

Queremos aquí subrayar que la escuela, como escenario socializador, constituye también un espacio donde se manifiestan las diferentes expresiones culturales de la sociedad, por lo tanto, ella reproduce igualmente los estereotipos que sobre los géneros perviven en el imaginario colectivo. Contribuir a interrumpir la cadena que sustenta la diferencia mediante la comprensión de los factores que la perpetúan, al igual que la promoción de las relaciones equitativas e igualitarias, es una tarea que corresponde a la actual escuela. Acerca de ello, expresan dos especialistas:

Una educación no sexista implica ser cuidadosas (os) en derrotar los sexismos en el currículo oculto y esto implica revisar el lenguaje en la educación y fuera de ella; tener presente que los materiales y contenidos educativos promuevan igualdad de derechos y oportunidades para mujeres y hombres, formar a maestros y maestras con una perspectiva de género, garantizar una asistencia de planeación en género, desarrollar el proyecto de educación sexual, lanzar tareas desde la pedagogía, llevando a revisar los PEI de cada institución escolar, los textos utilizados en la escuela y su contenido y todas las relaciones de género que se desarrollen en el ambiente escolar (Herrera y Morales, 2009, p.45).

Al respecto, Castro (2006) afirma:

La convivencia se aprende, se va construyendo, y la escuela puede y debe constituirse en lugar idóneo para que los alumnos aprendan las actitudes y conductas básicas de la convivencia y los docentes se realicen personal y profesionalmente, pero, sobre todo, debe ser un espacio donde personas distintas, con intereses diferentes, puedan encontrarse y estar bien (p. 13).

De acuerdo con el autor de esta cita, la escuela debe ser el espacio propicio para construir la paz, el amor, la participación, la tolerancia, el respeto, la democracia, las relaciones de amistad; y no la violencia, ni la agresividad. En relación con esto, si se está de acuerdo que la escuela es el espacio ideal para que los educandos logren sus aprendizajes básicos, se requiere reconocer en cada uno de ellos sus potencialidades, habilidades y destrezas. Ellos indicarán las mejores maneras de lograr esos objetivos o logros planteados. Al trabajar sobre las diferencias, se logrará que, por senderos diversos, cada uno de los estudiantes llegue a 
configurar su personalidad, sin perder de vista que todo esto es posible si hay interacción social.

En muchas ocasiones el profesor es el modelo, el espejo o el ejemplo a seguir de sus alumnos. Por ello,

los profesores, preparados casi en exclusiva para transmitir saberes, se ven necesitados de otro tipo de formación para poder no solo instruir a su alumnado sobre los planes de estudios, sino socializarlos para nuevas formas de ciudadanía (Loscertales y Núñez, 2009, p.8).

Sin embargo, hoy en las escuelas ya no solo se habla de las agresiones y actos de violencia que se generan entre alumnos, entre profesores y alumnos, sino también de la agresión entre los mismos docentes, bien sea por situaciones ajenas al quehacer pedagógico o por cuestiones personales o meramente académicas. Por ejemplo, cuando un profesor es tutor de un grupo y hay otro docente del mismo grupo que presenta métodos poco ortodoxos con sus pupilos, ya sea porque los agrede verbalmente, los humilla delante de sus compañeros, les exige tareas imposibles de realizar o los amedranta, entre otros comportamientos, se puede generar un conflicto porque el docente tutor reclama al docente agresor su inadecuada conducta hacia sus alumnos-pupilos, creándose en ocasiones enemistad entre los profesores. También existen otras situaciones, donde son los alumnos quienes ponen en riesgo la relación entre sus maestros, puesto que comentan lo que les dice su director de grupo al otro docente para crear conflictos y rencillas entre ellos, y de esa manera se vengan de su agresor.

De ahí la gran importancia del comportamiento del docente, si éste presenta un comportamiento agresivo, no solo con los estudiantes, sino con sus compañeros; mostrando una actitud prepotente, un discurso contestatario, si ejerce presión y es coercitivo; en consecuencia, es muy posible que sus estudiantes asimilen estas actuaciones equivocadas y desatinadas. El educador puede, con su actitud llegar incluso, a poner a sus educandos en contra de $\mathrm{X}$ o Y profesor, o a través de habladurías acerca de un colega puede generar un ambiente escolar de hostilidad, violencia e intolerancia; entonces: ¿quién enseña a quién?, ¿qué enseña el docente?, ¿qué aprende el estudiante en la escuela?, ¿se forma o deforma el educando en la escuela? 
En consonancia con lo anterior, se debe señalar que, a nivel nacional, desde el 15 de marzo del año 2013, se implementó la Ley 1620, por la cual se crea el Sistema Nacional de Convivencia Escolar y Formación para el Ejercicio de los Derechos Humanos, Sexuales y Reproductivos y la Prevención y Mitigación de la Violencia Escolar, con el fin de señalar una ruta única de información para reportar los casos de abuso escolar, embarazos en menores y violencia, y así lograr una respuesta efectiva ante la problemática.

El artículo primero de dicha ley, señala que el objeto de la misma, es

[...] contribuir a la formación de ciudadanos activos que aporten a la construcción de una sociedad democrática, participativa, pluralista e intercultural, en concordancia con el mandato constitucional y la Ley General de Educación -Ley 115 de 1994-mediante la creación del sistema nacional de convivencia escolar y formación para los derechos humanos, la educación para la sexualidad y la prevención y mitigación de la violencia escolar, que promueva y fortalezca la formación ciudadana y el ejercicio de los derechos humanos, sexuales y reproductivos de los estudiantes, de los niveles educativos de preescolar, básica y media y prevenga y mitigue la violencia escolar y el embarazo en la adolescencia.

Es muy importante la norma citada, porque la educación es un derecho fundamental de los niños, y un servicio público esencial del Estado, y no es coherente con tal precepto que muchos niños pierdan el interés en la educación, por causa de una situación que, manejada adecuadamente, dejaría de existir. La ley 1620 de 2013 busca contribuir, entre otros aspectos, a la formación de ciudadanos que puedan aportar a la construcción de una sociedad donde la convivencia pacífica sea posible, y donde, a su vez, se promueva y fortalezca la formación ciudadana y el ejercicio de los derechos humanos, sexuales y reproductivos de los estudiantes, por ello en esta ley se definen de manera muy precisa algunos conceptos claves que sirven para la construcción y entendimiento de los manuales de convivencia y para el caso que nos interesa: el currículo, al dejar claro los conceptos relacionados con la formación de los estudiantes. En el artículo $2^{\circ}$ la ley define un concepto muy importante para la formación de niños, niñas, adolescentes y jóvenes se trata del concepto de "Competencias ciudadanas", y señala que "Es una de las competencias básicas que se define como el conjunto de conocimientos y de habilidades cognitivas, emocionales y comunicativas que, 
articulados entre sí, hacen posible que el ciudadano actúe de manera constructiva en una sociedad democrática".

En Colombia, este tema ha interesado desde hace mucho tiempo a investigadores (Parra y otros 1992). En el estudio llamado La escuela violenta, se realiza un análisis del problema de la formación ciudadana en la escuela, es decir, se analiza la manera cómo esta institución cumple su papel de formar individuos para la participación activa, pacífica, democrática y justa en la sociedad civil. Se espera que la escuela, además de transmitir, distribuir y crear conocimiento, esté en capacidad de proporcionar una formación en valores para la convivencia en la sociedad. Se señala que es a estos dos objetivos donde debe apuntar la calidad de la educación.

Este es, sin duda, uno de los retos que tiene el sistema educativo del país, es decir el desarrollo e interiorización de las competencias ciudadanas en los alumnos y la comunidad educativa en general se constituye en factor fundamental para la formación integral de los estudiantes. La escuela, ya hemos señalado, tiene la responsabilidad de formar ciudadanos libres, partícipes responsables de la vida en sociedad, pensantes y críticos, que hagan posible la cohesión social, tal como está indicado en el artículo $5^{\circ}$ de la ley 115 de febrero 8 de 1994, o Ley General de Educación, que señala que el fin de la educación lo constituye "la formación en el respeto a la vida y a los demás derechos humanos, a la paz, a los principios democráticos, de convivencia, pluralismo, justicia, solidaridad y equidad, así como el ejercicio de la tolerancia y de la libertad".

Para Uruñuela (2008), uno de los retos mayores del sistema educativo, junto con la transmisión de conocimiento, es lograr que los estudiantes aprendan a convivir como personas y como ciudadanos en el mundo actual. Así, define la educación para la ciudadanía como

[...] el conjunto de prácticas y actividades diseñadas para ayudar a todas las personas, niños, jóvenes y adultos a participar de manera activa en la vida democrática, aceptando y practicando sus derechos y responsabilidades en la sociedad. Implica, por tanto, el desarrollo en los alumnos de las competencias necesarias para relacionarse adecuadamente con el mundo que les rodea y la puesta en marcha de acciones colectivas para lograr una vida mejor para todos (p.42).

En Colombia, el Ministerio de Educación ha mostrado su interés y preocupación por el tema creando el Programa de Competencias Ciudadanas, como una estrategia para el logro 
de una educación de calidad. Este programa, desde sus inicios, señaló que se orientaría a desarrollar las habilidades, destrezas y conocimientos sobre ciudadanía y convivencia en los estudiantes de todos los rincones del país. El texto indica:

El Programa de Competencias Ciudadanas - $\mathrm{PCC}$ - es el conjunto de estrategias lideradas desde el Ministerio de Educación Nacional - $\mathrm{MEN}$ - y dirigidas a todo el sector, que busca fomentar en el establecimiento educativo innovaciones curriculares y pedagógicas basadas en "prácticas democráticas para el aprendizaje de los principios y valores de la participación ciudadana" (Art. 41, Colombia, 1991), con el fin de "formar al colombiano en el respeto a los derechos humanos, a la paz y a la democracia (Art. 67, Ibíd.).

Sin lugar a dudas, la formación para la ciudadanía implica más que el conocimiento de tales competencias, pues debe procurar el desarrollo de habilidades, actitudes y acciones que hagan posible la convivencia, la paz y la participación democrática. Conocer la Constitución Nacional, los mecanismos de participación y las prácticas democráticas constituyen eventos muy importantes para la formación integral de los jóvenes, pero estos conocimientos deben servirle especialmente para adoptar un comportamiento ciudadano y, en las instituciones educativas, esta formación debe de realizarse de forma transversal, incluyendo todas las áreas, pues en todas se puede procurar el aprendizaje del trabajo colectivo, la toma de decisiones colectivas justas, el respeto por los derechos humanos, el respeto por la diferencias culturales, sociales y de género. Así mismo, las distintas instancias de la institución escolar o comunidad educativa —entendiendo las directivas, el cuerpo docente, la asociación de padres - deben estar involucradas para lograrlo mediante las distintas estrategias y actividades.

\section{El papel de la familia frente a la escuela}

Sin lugar a dudas, la familia es el elemento más importante del medio donde vive el niño, pues ella juega un papel esencial en el desarrollo de su personalidad, así como en su comportamiento. En nuestro medio, sin negar las mutaciones y los cambios que también ha sufrido la familia tradicional, esta institución se tiene como el principal agente educativo en el proceso de socialización del individuo. Es en este grupo primario donde se inicia y se refuerza ese proceso mediante el cual los seres humanos se apropian, aprenden e incorporan los valores, las normas y comportamientos necesarios para la vida en sociedad. 
Al interior de la familia, se plantea también el problema de la prevención o la facilidad de la conducta violenta y/o la conducta desviada. Se ha afirmado, incluso, que ciertos jóvenes cometen hechos que la ley califica como delitos, generalmente hurtos y robos con violencia sobre las cosas, por actitudes de contraposición a su familia y a las reglas sociales; otros, en cambio, responden a los mensajes recibidos en el seno de la familia o de su medio social.

Se ha llegado a afirma que "el menor que transgrede en forma repetida las normas sociales se constituye en un síntoma de la dificultad familiar y social para establecer, trasmitir, manejar y asumir las normas" (Jiménez, 1998, p.9). Por esta razón, para comprender las dificultades del menor de edad para apropiarse de los valores, normas y comportamientos requeridos para convivir en sociedad, es necesario explorar temas relativos a la dinámica familiar, el manejo de la autoridad, la normatividad y las condiciones de socialización. Muchas investigaciones se han realizado para tratar de establecer los factores familiares que pueden llevar a un joven, a infringir las normas y leyes de la convivencia y la sociedad.

\section{Factores familiares señalados como determinantes del comportamiento violento de niñas, niños y adolescentes}

En relación con el papel de la familia en el problema de la violencia escolar Olwes (1999), señala en su estudio cuatro factores familiares como determinantes de la agresividad de los niños: <<falta de ternura, laxismo parental, métodos de educación represivos (castigos corporales y crisis emocionales violentas) y el temperamento del niño (activo o calmo) >>. Así, una falta de amor y de atención y un exceso de libertad en el transcurso de la infancia son condiciones que contribuyen fuertemente al desarrollo de un modo de reacción agresiva. Efectivamente, las malas relaciones familiares han sido señaladas por otros estudios (Cusson 1990) que indican la importancia de fuertes lazos en la familia con el fin de que el niño o el adolescente pueda encontrar en su círculo familiar el afecto, la estabilidad y la autoridad que necesita. Los estudios convergen en la idea de que los jóvenes delincuentes y los jóvenes violentos rara vez tienen buenas relaciones con sus padres, estos, a su vez, se manifiestan con frecuencia con frialdad y hasta hostilidad hacia sus hijos; por otro lado, los hijos interrogados acerca de sus padres manifiestan que no los aprecian (especialmente al padre más que a la madre) que no quieren parecérseles y que se comunican poco con estos. 
Las carencias educativas de los padres: Es otro de los factores señalados en el comportamiento problemático de los adolescentes, se trata aquí de aspectos concernientes más bien relacionados con la personalidad de los padres. Con frecuencia los progenitores de los jóvenes con problemas de conducta han sido caracterizados como adultos débiles, pasivos, que pasan por alto faltas serias de sus hijos y alternan sus reacciones entre la complacencia y el castigo severo a los hijos. Acerca de ello,

cuando los padres justifican permanentemente las faltas de sus hijos, cuando ante una transgresión de la norma, unas veces sancionan y otras no, o, lo que es peor, amenazan, pero no cumplen con los castigos, crean condiciones para que los hijos no respeten las normas. En los casos extremos, el menor sabe o deduce que no va ser sancionado por su comportamiento, cualquiera que sea (Jiménez, 1998, p.14).

Las prácticas educativas parentales: Entendidas como el conjunto de conductas conscientes o inconscientes de los padres, susceptibles de afectar al adolescente en el plan psicológico y particularmente afectivo. Ellos comprenden las palabras y los comportamientos dominantes de los padres en la interacción con los hijos. Si las prácticas educativas de los padres son desvalorizantes o negativas, la representación o la imagen que el niño tendrá de sí mismo será también negativa y eso va a influir en su comportamiento.

La disociación del grupo familiar: Igualmente ha sido señalada como una variable que puede tener fuertes repercusiones en el comportamiento del niño o joven.

En las investigaciones relativas al papel de las familias disociadas en la formación y manifestación del comportamiento delictivo o problemático del joven, el divorcio disociación familiar "primaria" con frecuencia es el elemento explicativo de la aparición de tal conducta. Sin embargo, ciertos estudios (Koudon, 1989) han puesto de relieve la importancia de la llamada disociación familiar "secundaria". En esta, parámetros diferentes al divorcio han sido tenidos en cuenta; ya no es la presencia o ausencia de uno u otro miembro de la familia lo que importa, sino la forma como en el seno de la familia las relaciones entre sus miembros se desarrollan; se ha concluido que la ruptura en un sentido físico no significa, ipso facto ruptura de las relaciones entre los padres, no significa tampoco que automáticamente haya contradicción entre ellos. 
La ruptura física conyugal no es siempre mal vivida por lo niños y adolescentes, ya que puede haber ruptura, aunque físicamente los padres estén juntos. Entonces la presencia o la ausencia física de los padres en sí misma no es significativa; lo que es revelador es el lugar que cada uno de los padres concede al otro en su discurso o su comportamiento y el lugar que confieren al niño.

Las carencias afectivas: Es, así mismo, un factor difícil de aprehender, sin embargo algunos autores afirman que él juega un papel considerable entre los miembros de las bandas. Ellos ven en el ingreso del joven a una banda una especie de compensación de una carencia afectiva, aunque se precisa que por lo general los lazos afectivos en una banda son de tipo horizontal e igualitario y en consecuencia diferente a los que se establecen entre padres e hijos en el seno de una familia.

El rechazo parental: Es aquel de los padres hacia los hijos, definido de varias maneras: falta de amor, carencia de afecto, etc. Pero el concepto unificado es que esos términos son el reflejo del aprecio o de las actitudes de los padres hacia sus hijos. Con frecuencia tanto la psicología como la sociología lo han asociado a la delincuencia juvenil y al comportamiento agresivo del joven. Se ha dicho que el rechazo puede ser la causa, pero también la consecuencia de esos comportamientos en los jóvenes. Efectivamente el rechazo de los padres puede conducir a los hijos a rebelarse, pero igualmente debe admitirse que puede hacérsele difícil a un padre amar a un hijo rebelde. Sin lugar a dudas, padres indiferentes hacia sus hijos, estarán menos dispuestos a brindar una buena y constructiva vigilancia, necesaria para el desarrollo armonioso de niños y adolescentes.

Ahora bien, en torno al tema del acoso escolar, se pretende que para contrarrestar este fenómeno participen activamente no solo la escuela, como la institución que contribuye a generar cambios, comportamientos, valores, cultura y conocimientos, es decir, formación integral, sino también la familia, la sociedad y el Estado, en concordancia con la ley 1098 de 2006, que estipula el principio constitucional de la corresponsabilidad entre estas instituciones. Igualmente, el decreto No. 1286 del 27 de abril de 2005, que establece normas sobre la participación de los padres de familia en el mejoramiento de los procesos educativos de los establecimientos oficiales y privados. 
Referente al papel de la familia en esta problemática, ya se ha señalado cómo esta es el espacio donde se forma el individuo (niño-niña) a partir de los ejemplos, experiencias y aspectos socioculturales que experimenta como resultado de interacción social, es decir, es allí donde empieza el proceso de sociabilidad de las personas. Y a ella le cabe la responsabilidad de asumir la orientación de las nuevas generaciones para cimentar desde temprana edad principios y valores que contribuyan a configurar su personalidad.

En la escuela, los jóvenes interactúan con otros jóvenes de su grupo social, y es precisamente allí donde ellos transforman la educación recibida en el núcleo familiar, para extrapolarla, de manera inconsciente, en muchos casos, a las costumbres y estilos de vida de los otros individuos de ese grupo. Al respecto, Díaz, Zariñana y Rodríguez (2015) afirman que el niño y la niña deben ir aprendiendo dónde se encuentran los límites de lo que se considera conducta agresiva con el resto de la gente. En muchos casos la familia hoy parece dejar la educación y la formación de valores de sus hijos a la escuela, delegando o trasladando esa responsabilidad a la institución. La familia hace parte de la comunidad. La comunidad influye en la calidad de vida del grupo familiar, por eso es necesario que los integrantes de la familia se involucren en las diferentes actividades de la comunidad, comprendida la escuela.

\section{Resultados}

En la cartilla para padres de familia (Guías No. 26, MEN) se preguntan sobre ¿cómo participar en los procesos educativos de la escuela? Contextualizan la problemática de los padres con respecto a la responsabilidad de estos con la formación de sus hijos (as):

La velocidad del mundo moderno ha obligado a que los padres deban salir del hogar para responder económicamente por la familia; este hecho ha generado que el tiempo para compartir con los hijos sea mínimo. Como consecuencia de este fenómeno, la escuela se debería convertir en ese espacio que vincule familia, afecto, formación y conocimiento. Las contradicciones arriba mencionadas, además de ser frecuentes entre las familias, generan en los niños desconcierto en lo relacionado con su mundo inmediato y posiblemente dificultades en su desarrollo emocional y personal (p.7).

De la misma manera, las familias disfuncionales y la desconfiguración de sus funciones han hecho que las instituciones educativas reciban muchos jóvenes que padecen problemas afectivos, emocionales y con múltiples carencias que van en detrimento de la convivencia. 
Para el caso de Colombia, hay que tener en cuenta también el contexto sociopolítico de violencia en que ha vivido el país y los problemas de inseguridad, delincuencia y violencia cotidianas, distintas formas de violencia, organizadas y no organizadas que van incidiendo en el estado psíquico del joven, y que de una u otra forma esa situación de violencia se va a reflejar en la escuela. La agresividad escolar es, a veces, una prolongación de la violencia de la propia sociedad manifestada en la calle. Existe una fuerte relación entre ambas y podemos considerar a la violencia escolar como un caso particular de la violencia general. Por ello, interviniendo en la escuela, se reportarán beneficios a la comunidad (Andershed, Kerr y Stattin, 2001).

Al respecto Berdondini (1996) y Gibbs y Sinclair (1999) consideran que también la familia, cuando está desestructurada, puede ser el elemento clave en las génesis de las conductas agresivas. En el mismo sentido, Junger (1996) afirma que el bullying proviene de familias con problemas, de padres erráticos y del uso de métodos disciplinarios duros y bruscos.

En un estudio, Vega (2007), realizado en la comunidad de la Institución Educativa Distrital San Francisco Javier, de la ciudad de Santa Marta, y la cual se propuso analizar las relaciones existentes entre violencia intrafamiliar con el aprendizaje, encontró que su impacto es altamente significativo en el sentido de que la violencia en la familia provoca dificultades en las relaciones interpersonales y bajo rendimiento académico. Vega, afirma:

[...] los niños han transferido automáticamente al ámbito escolar los problemas de violencia con los que conviven; lo cual significa que han incorporado estos patrones a los escenarios donde se relacionan con sus pares. Estas experiencias van creando en la mente del niño modelos de relaciones basadas en la idea de que es normal resolver conflictos de esa manera, y entonces lo replica en la escuela y, por esto, el padre se sorprende de que su conducta merezca alguna sanción y repita lo que su hijo le dice que los profesores y compañeros lo acosan y maltratan. Como se pudo apreciar en las actitudes de algunas madres y padres, era normal que el niño se defendiera de los ataques "supuestamente" propiciados por sus compañeros e incluso, sus profesores. (p.1).

En concordancia con lo anterior, en el artículo 67 de la Constitución Política de Colombia se dispone: "El Estado, la sociedad y la familia son responsables de la educación, que será obligatoria entre los cinco y los quince años de edad y que comprenderá como mínimo, un 
año de preescolar y nueve de educación básica". En esta cita se establece con claridad la responsabilidad de la familia en la formación y educación de sus hijos (as).

Expresado de otra manera, las instituciones educativas se deben convertir en el espacio natural donde los padres o personas responsables de los niños y jóvenes, además de confiar la educación y formación integral de éstos, encuentren la oportunidad de reflexionar sobre la forma como se desempeñan como padres y la manera como pueden ser parte activa en la formación de los hijos, en la medida que establezcan una relación de corresponsabilidad en la formación y construcción de valores con directivos, docentes, con otros padres de familia, $\mathrm{y}$, en general, con toda la comunidad educativa en beneficio de los niños y niñas.

No obstante, la realidad dista mucho de lo que arriba se dice. No es cierto que las instituciones oficiales, involucren o hagan partícipe a los padres de familia de todas las actividades que realiza la escuela; por ejemplo, cuando se conforma el gobierno escolar, cuando se actualiza o ajusta el manual de convivencia, se toma una decisión en torno a algún conflicto o acto de violencia (bullying) sucedido al interior del colegio, o hay comisión de evaluación.

En todo caso, los padres deben y quieren hacer parte activa de la formación de sus hijos, a través de las diferentes acciones que los puedan involucrar, y en la que ellos puedan ser parte fundamental en la solución de algún conflicto, y en muchos casos no se les permite.

En cambio, si la escuela permite la participación activa de los padres de familia, en lo que concierne a ellos, sin que estos se extralimiten, ellos, tal vez, podrían ser sujetos dinamizadores y responsables de lo que hacen y saben los estudiantes.

\section{Conclusiones}

Generalmente, se ha aceptado que la escuela es una institución social que se basa en las relaciones interpersonales y la socialización del ser humano y de todos los miembros de la comunidad académica; se reconoce, así mismo, la importancia y el rol que desempeña la escuela en la protección de los niños, niñas y adolescentes contra la violencia, especialmente el maltrato infantil y el abuso sexual al interior de las familias, sin embargo una constatación es evidente: el espacio escolar también puede constituir un entorno donde el joven se ve expuesto a la violencia. 
Por otro lado, se debe señalar que todo lo que se refiera a convivencia dentro de las escuelas debe compadecerse con la misión, visión y modelo pedagógico y ser coherente con el currículo y el contexto de estas. Por esta razón, es pertinente que se plantee una estrategia adecuada de trabajo para afrontar la resolución de los conflictos de modo que contribuya a la formación de los educandos en habilidades sociales que posteriormente, en su vida adulta, les permita ser ciudadanos que frente a cualquier conflicto posean herramientas, habilidades y competencias para su resolución pacífica. Así mismo, la escuela transmite a los estudiantes otros contenidos que, aunque no aparecen explícitamente en los documentos oficiales, van a marcarlo. Entre ellos, se encuentra el currículum oculto o latente, que forma básicamente en valores y actitudes y que estará influenciado por la ideología del grupo social dominante en la institución educativa de que se trate. De hecho, este currículum oculto puede llegar a estar en contraposición con el currículo oficial, y con frecuencia las prácticas institucionales y personales que lo constituyen pueden ser mucho más sutiles y efectivas que las del currículum explícito (Sandoval, M., 2014).

En el país, con la ley 1620 de 2013, el Estado entró a reforzar lo dispuesto en la Ley General de Educación o ley 115 de febrero 8 de 1994, que en el artículo $5^{\circ}$ señala los fines de la educación y particularmente en el inciso $2^{\circ}$ de dicho artículo, plantea que uno de esos fines es "la formación en el respeto a la vida y a los demás derechos humanos, a la paz, a los principios democráticos, de convivencia, pluralismo, justicia, solidaridad y equidad, así como el ejercicio de la tolerancia y de la libertad". Las escuelas deben ser, pues, espacios adecuados para el aprendizaje de la convivencia en el marco de una democracia.

La racionalidad de la acción comunicativa se refiere a la interacción de a lo menos dos sujetos capaces de lenguaje y de acción que entablan una relación interpersonal. Los actores buscan entenderse sobre una situación de acción para poder así coordinar de común acuerdo y establecer la ruta integral para la convivencia. Que, además, y esto es muy importante, "nos permitirá evitar la deserción escolar generada, en parte, por los conflictos relacionados con el acoso escolar, la agresión física y la agresión verbal” (Campo, 2011, p. 25) pues el maltrato ejercido contra los niños(as) tiene como una de sus consecuencias la pérdida del potencial humano manifestada con frecuencia en la disminución de su capacidad para aprender en los primeros años de educación. 
En cuanto al papel de la familia en la problemática de la convivencia y violencia en la escuela, bien ha manifestado el Ministerio de Educación la necesidad de la participación de los padres de familia en las escuelas para lograr una educación de calidad, pues es responsabilidad de las instituciones educativas fortalecer el rol de éstos, como formadores y participantes activos del proceso de formación de sus hijos.

Las instituciones educativas deben implementar estrategias para permitir e incentivar la participación de los padres y la manera como pueden ser parte activa en la formación de los hijos, ello en razón del principio de corresponsabilidad que debe guiar todos los procesos relacionados con los niños, niñas y adolescentes, en este caso la formación y construcción de valores realizadas en la escuela. 


\section{Referencias}

Andershed, M; Kerr, M y Stattin, (2001). Bullying in School and Violence on the Streets: Are the Same People. Journal of Scandinavian Studies in Criminology And Crime Prevention.vol.2.

Arias, S. L. (2019). Conocimiento didáctico del contenido de la enseñanza de ética y valores humanos, un estudio de caso. Cedotic 4. (2). Consultado en:

http://investigaciones.uniatlantico.edu.co/revistas/index.php/CEDOTIC/article/view/2363/2907

Arnaz, J. (1995) La planeación curricular. México DF: Ed. Trillas

Berdondini, L (1996). Cohesion and Power in the Families of Children in Bullying/Victim Problems at School: An Italian Replication. . Firenza-Italia: Univ. degli Studi di Firenze. Departamento di Psicologia. Firenze.

Busino, G. (1979). Idées et Faits. Revue Européenne des Sciences Sociales, tome XVII, No. 48.

Castro, A (2006). Violencia silenciosa en la escuela. Dinámica del acoso escolar y laboral. Buenos Aires-Argentina- Editorial Bonum.

Carrillo, B. (2009) importancia del currículo oculto en el proceso de enseñanza-aprendizaje. Disponible en:

https://archivos.csif.es/archivos/andalucia/ensenanza/revistas/csicsif/revista/pdf/Numero_1 4/BEATRIZ_CARRILLO_2.pdf

Chaux, E; LLERAS, J y VELÁSQUEZ, A. (2004). Competencias ciudadanas: de los estándares al aula. Una propuesta de integración a las áreas académicas. Recuperado de: http://www.colombiaaprende.edu.co/html/mediateca/1607/articles-75077_archivo.pdf

Chaux, E. (2012). Educación convivencia y agresión escolar. Bogotá D.C. Colombia: Aguilar, Altea, Taurus, Alfaguara. Primera edición.

Cusson, M (1990). Croissance et décroissance du crime. Ed.Puf. Paris

Díaz-Aguado, M. (2004). Del acoso escolar a la cooperación en las aulas. Madrid, España: Pearson. 
Díaz-Barriga, F (2008). Educación y nuevas tecnologías de la información: ¿Hacia un paradigma educativo innovador? Revista Electrónica Sinéctica, núm. 30, 2008, pp. 1-15 Instituto Tecnológico y de Estudios Superiores de Occidente Jalisco, México.

Díaz-Barriga, F., et al. (1990) Metodología de Diseño Curricular para la Educación Superior. México. Trillas. Disponible en http://dip.una.edu.ve/mpe/020dise\%C3\%B1ocurricular/lecturas/Unidad_III/Metodologia_d e_Disenno_Curricular_Unidad_III.pdf

Díaz, D; ZARIÑANA, R. y Rodríguez, D. (2015) Acoso escolar: Una mirada desde el contexto familiar. En Bullying. Estampas infantiles de la violencia escolar: Exploraciones psicológicas (compilación: Mario Orozco Guzmán, Ana María Méndez Puga, Yolanda Elena García). Sonora-México: Editorial El Manual moderno, S.A de C.V.

Fernández, I. (2011). Guía para la convivencia en el aula. España, S.A: Wolters Kluwer. $4^{\mathrm{a}}$ edición.

Frechette y Le Blanc, (1987). Délinquence et délinquants. Gaêtan Morin, chicoutimo. Québec.

Jiménez-Zuluaga, B (1998). La familia y el menor infractor. Cuadernos Familia, cultura y sociedad No. 2, colección Cuadernos del CISH, Universidad de Antioquia, noviembre de 1998, Medellín, Colombia.

Gibbs. I. y SINCLAIR, I. (4,1 feb 1.999). Treatment and Treatment Outcomes in Children's. Child and Family Social Work, 1-8.

Herrera, E y Morales, H. (2009) El reto de la inclusión de la perspectiva de género en los currículos. Justicia Juris, Vol 8, pp. 45-50.

Hoyos, O., Aparicio, J. \& Córdoba, P. (2005) Caracterización del maltrato entre iguales en una muestra de colegios de Barranquilla (Colombia). Revista Psicología desde el Caribe 16, 1- 28.

Johnson, M (1967) La teoría del currículo. Educational Theory. 1967 ; 17 (2) :12-24.

Junger, T. J. (1.996). Youth and violence in Europe, en Studies on Crime and Crime Prevention. Norway Scandinsvia: Univ.Press.

Koudon, O. (1989). Familles dissociès “secondaires”. En : Cöte divoire et comportements 
délinquants des adolescents. In : Revue International de Criminologie et de police technique, Vol. 47, No.2 Paris, 1989.

Koudon, O. (1993). Pratiques éducatives parentales et identité négative chez les adolescents inadaptés en Côte d'Ivoire. Revue Internationale de Criminologie et de Police Technique, Vol. 46 No.3, 345-358.

Loscertales, F y NÚÑEZ, T (2009). Violencia en las aulas. El cine como espejo social. Barcelona-España- Octaedro.

Loeber, R. \& Stouthamaer, M. (1986). Family Factors as Correlates and Predictors of Juvenile Conduct Problems and Delinquency. Crime and Justice, Vol.7, University of Chicago Press, Chicago.

Magendzo, A. (2003) Currículum, convivencia escolar y calidad educativa. Disponible en https://www.oei.es/historico/valores2/monografias/monografia02/reflexion03.htm.

Martínez, J. (2014). El manual de convivencia y la prevención del bullying. Diagnóstico, estrategias y recomendaciones. Bogotá D.C., Colombia: Cooperativa Editorial Magisterio. $1^{a}$ edición.

MINISTERIO DE EDUCACIÓN NACIONAL, (2006). ¿Cómo participar en los procesos educativos de la escuela? Guías No. 26. Cartilla para padres de familia. En Revista Revolución educativa. Disponible en: https://www.mineducacion.gov.co/cvn/1665/articles$\underline{120646 \text { archivo_pdf.pdf }}$

MINISTERIO DE EDUCACIÓN NACIONAL DE COLOMBIA. Ley General de Educación de Colombia (1994). Tomado de: http://www.oei.es/quipu/colombia/Ley_115_1994.pdf

Morales, H. y Vásquez, R. (2014). Conflicto, violencia y mediación en los espacios educativos. Barranquilla, Colombia: Uniautónoma.

Olweus, Dan. (1999) Violences Entre élèves, Harcèlements Et Brutalités. Les faits, les solutions. Paris. ESF.

Parra-Sandoval, R; GONZÁLEZ, A; Moritz, O; Blandón, A. y Bustamante, R. (1992). La escuela violenta. Bogotá: Fundación FES-Tercer Mundo Editores.

Pérez, T (2014) El poder transformador de los educadores. Reflexiones y herramientas para cultivarlo. Bogotá D.C., Colombia: Cooperativa Editorial Magisterio. 
REPÚBLICA DE COLOMBIA. Ley 1098 de 2006.

REPÚBLICA DE COLOMBIA. Ley 1620 de 2013

Sandoval, M. (2014) Convivencia y clima escolar: claves de la gestión del conocimiento Última Década, núm. 41, diciembre, 2014. Centro de Estudios Sociales Valparaíso, Chile.

Tyler, R (1973) Principios básicos del currículo. Buenos Aires: Editorial Troquel .

Vega, L. (2007). La violencia intrafamiliar y el aprendizaje en la escuela: Un estudio etnográfico en la Institución Educativa Distrital (I.E.D.) San Francisco Javier Santa Marta Disponible en: https://www.monografias.com/trabajos71/violencia-intrafamiliaraprendizaje-escuela/violencia-intrafamiliar-aprendizaje-escuela2.shtml 